\title{
High-field thermal noise of holes in silicon: The effect of valence band anisotropy
}

\author{
J. M. Hinckley a) and J. Singh \\ Department of Electrical Engineering and Computer Science, The University of Michigan, Ann Arbor, \\ Michigan 48109-2122
}

(Received 3 June 1996; accepted for publication 5 September 1996)

\begin{abstract}
The effects of valence band anisotropy on longitudinal and transverse high-field differential mobilities, diffusivities, and thermal noise temperatures were theoretically investigated. The effects were examined for holes in silicon and in several hypothetical materials having systematically varied degrees of valence band anisotropy. The results show a pronounced dependence of the transverse high-field differential mobility and of the longitudinal high-field noise temperature upon the degree of anisotropy. This suggests that thermal noise measurements may provide an alternative to magnetotransport methods as a valence bandstructure measurement technique. The results also imply that thermal noise in semiconductor devices can be mitigated by choosing a semiconductor with a low degree of valence band anisotropy and, for a given semiconductor, by properly aligning the device layout with respect to the material crystallographic axes. (C) 1996 American Institute of Physics. [S0021-8979(96)03124-6]
\end{abstract}

\section{INTRODUCTION}

The subject of this article is the effect of valence band anisotropy on the high-field thermal noise characteristics of holes in silicon. Investigation was carried out theoretically by using a Monte Carlo technique to simulate the dynamics of holes, under a high electric field, over a range of semiconductor materials. In addition to silicon, the other materials studied, which were hypothetical, differed from silicon only through a systematic variation of their Luttinger valence band structure parameters. ${ }^{1}$ This variation was to control the degree of valence band anisotropy while leaving all other aspects of the semiconductor material model the same as that of silicon. The variation of the noise characteristics, extracted from the Monte Carlo output, was related to the variation of the band structure parameters and, thereby, to the system's degree of anisotropy.

The motivation for studying the effect of the valence band anisotropy on high-field hole thermal noise is twofold. First, it provides information for the control of thermal noise in a device through the choice of the semiconductor material and through the crystallographic orientation of the material with respect to the biasing electric field in the device. Second, it suggests the use of high-field hole noise characteristics as a probe of the details of the semiconductor valence band structure.

In this second area, the anisotropic nature of semiconductor valence bands can be quantified by the material's Luttinger valence band parameters. ${ }^{1}$ The Luttinger parameters, in principle, may be determined by magnetotransport measurements. ${ }^{2,3}$ However, in practice, it is difficult to measure the Luttinger parameters in this way because very high magnetic fields are required. A simpler transport measurement that could yield the Luttinger parameters would be very useful. Many hole transport measurements that do not involve magnetic fields, such as velocity-field measurements,

${ }^{\text {a)} E l e c t r o n i c ~ m a i l: ~ n c k o @ e n g i n . u m i c h . e d u ~}$ have negligible dependence on the anisotropy of the valence band, and are primarily controlled by the spatially averaged scattering rates. Thus, the density of states, and not the band anisotropy, determines such measurements. However, this investigation has found that hole thermal noise characteristics have a strong signature of the valence band anisotropy, and therefore, in principle, could yield information on the Luttinger parameters. A measurement based on noise properties would have a substantial advantage over magnetotransport methods since generation of a magnetic field would not be required.

Some types of noise in semiconductor devices, such as $1 / f$ and generation-recombination noise, may be controlled by the choice of device design and fabrication process. For example, the reduction of residual damage sites created in a fabrication process will reduce the amount of generationrecombination noise associated with such traps. However, thermal noise is generated by mechanisms that are intrinsic to the dynamic nature of the charge carriers, and therefore presents a lower noise limit in all semiconductor devices.

Although thermal noise, by its fundamental nature, is present in the operation of all types of semiconductor devices, one class of device in which it is particularly manifest is the field-effect transistor (FET). This is because the FET is essentially a voltage-controlled resistor. The scattering in the device channel that is characterized by this resistance gives rise to thermal noise. Under normal device biasing conditions, a high electric field is present in the channel, and highfield noise characteristics pertain. One means of controlling thermal noise in a device is to control the intrinsic resistance by using a semiconductor with a high carrier mobility. Another method used is to cool the device, thereby reducing the rate of carrier scattering. ${ }^{4}$

Aside from its aspect of interferring with the transmission of information through a system, thermal noise has been extensively studied as a probe of the microscopic processes that give rise to it. ${ }^{5-8}$ These investigations yield information both about the rates of different types of scatterings and 
about the semiconductor band structure. In the latter, most work has been concerned with features of the conduction band structure, such as intervalley separation and energy surface anisotropy.

Up until now, little work has been done to investigate the relationship between the shape of the valence band and the hole thermal noise characteristics. This is due, in part, to the complexity of rigorously modeling hole transport. However, there is reason to expect that the thermal noise characteristics of holes will exhibit features that reflect the valence band anisotropy. As will be discussed in Sec. II, a relationship exists between the mobility, the diffusivity, and the noise temperature. Both the high-field hole mobility and diffusivity have been studied with respect to the field orientation. ${ }^{9,10}$ In those works, both the transverse differential mobility and the diffusivity were found to vary significantly, depending on the orientation of the electric field in the high-field region. One would expect, therefore, that the high-field noise temperature, being essentially the ratio of diffusivity to mobility, would also exhibit a dependence on the field orientation. Since such a dependence would occur only for materials with an anisotropic band structure, the strength of the dependence should correlate to the degree of band structure anisotropy.

As the term is generally used, anisotropy of noise characteristics refers to the difference between the longitudinal and transverse characteristics for a single specified field orientation. Only sparse published information exists concerning the field-orientation dependence of any noise-related hole characteristics. In Ref. 5, experimental and theoretical silicon hole longitudinal diffusivity-field characteristics are presented for two field orientations: $\langle 100\rangle$ and $\langle 111\rangle$. These data do show a difference between the two orientations at $T=77 \mathrm{~K}$, but none at $T=300 \mathrm{~K}$. Aside from this and the references cited above, ${ }^{9,10}$ we are not aware of other work on the effect of valence band anisotropy on hole thermal noise characteristics.

\section{THEORY}

Noise in an electrical current is the random, nondeterministic fluctuation of that current. In semiconductor devices, important types of noise are $1 / f$, or flicker, noise, shot noise, generation-recombination noise, and thermal noise. ${ }^{11}$ Each of these affects the current flow, either by causing fluctuations in the number of flowing charge carriers or by causing fluctuations in the momentum and energy of the individual charge carriers.

Thermal noise, in particular, arises from fluctuations in the individual charge carrier's momentum and energy as a result of scattering. Such scattering, primarily phonon scattering in nonhighly doped semiconductors, may or may not be accompanied by a transfer of the charge carrier to a new valley or band. Where intervalley or interband scattering occurs, if the new state has a conductivity effective mass that is significantly different from that of the original state, the speed of the charge carrier will be affected, thereby contributing to the thermal noise. In the case of hole transport, although scattering does occur between the heavy hole, the light hole, and the split-off hole bands, the wide range of conductivity effective masses present in each of these bands obscures the identification of an interband noise component in the thermal noise. Therefore, in the discussion to follow, fluctuations in the hole current arising from both intraband and interband scattering will be termed thermal noise.

The Nyquist relationship establishes a connection between temperature and noise characteristics, namely,

$$
S_{i}=4 k T \operatorname{Re}(y),
$$

where $S_{i}$ is the current fluctuation spectral density, $\operatorname{Re}(y)$ is the real part of the small signal output admittance of the noise generator, $k$ is Boltzmann's constant, and $T$ is the temperature of the noise generator. It must be immediately stated that the Nyquist relation is valid only for a system that is in thermodynamic equilibrium with its environment. With the aid of heuristic circuitry, ${ }^{11}$ an expression for the maximum available noise power, $P_{\mathrm{av}}$, in a frequency interval $\Delta f$, may be derived from Nyquist's relation,

$$
P_{\mathrm{av}}=k T \Delta f \text {. }
$$

This gives the amount of noise power that can be delivered from a noise generator to an impedance matched load at temperature $T$. Again, this is valid only under conditions of thermodynamic equilibrium. When a system is biased, causing an electrical current to flow, the system is no longer in thermodynamic equilibrium. Under this condition, the maximum available noise power will differ from that given by Eq. (2). Replacing the temperature $T$ in Eq. (2) with a noise temperature $T_{n}$ serves to define the noise temperature so that, regardless of whether or not a system is in thermodynamic equilibrium, the maximum available noise power is given by

$$
P_{\mathrm{av}}=k T_{n} \Delta f .
$$

For thermal noise in semiconductors, $T_{n} \geqslant T$, being equal only under conditions of thermodynamic equilibrium.

By separately relating the current fluctuation spectral density to charge carrier velocity fluctuations and to the maximum available noise power, a relationship between the noise temperature and the carrier differential mobility, $\mu$, and diffusivity, $D$, may be established ${ }^{11}$ for uncorrelated particles. The relationship is

$$
\frac{k T_{n}}{q}=\frac{D}{\mu} .
$$

Under thermodynamic equilibrium, with $T_{n}=T$, this is the familiar Einstein relation. However, it remains valid under conditions of nonthermodynamic equilibrium, such as under a high electric field. In this case, the values of diffusivity and differential mobility are those of the particular high-field condition. For this relation between the transport parameters $\mu, D$, and the noise to be valid, carrier-carrier interactions must be negligible. This is because the relation between the current fluctuation spectral density and the diffusivity neglects any correlations between velocity fluctuations of different particles. Since the present work is not concerned with materials having high carrier densities, the effect of carrier-carrier correlations are neglected and Eq. (4) is used extensively. 
The Monte Carlo model used in this work has been fully documented in other publications, ${ }^{12-15}$ and will be only briefly described here. All three valence bands (heavy, light, and split-off hole) are included in the model. The band structure is calculated using the $\mathbf{k} \cdot \mathbf{p}$ method including spin-orbit coupling, which gives the full anisotropy of the energy surfaces. The energy values are obtained as the eigenvalues of a $6 \times 6$ Hamiltonian:

$$
H=H_{k p}+H_{s o} .
$$

The $\mathbf{k} \cdot \mathbf{p}$ Hamiltonian matrix is ${ }^{16}$

$$
H_{k p}=\left[\begin{array}{cc}
H^{\prime} & 0_{3 \times 3} \\
0_{3 \times 3} & H^{\prime}
\end{array}\right] \uparrow,
$$

$$
H^{\prime}=-\frac{\hbar^{2}}{2 m}\left[\begin{array}{ccc}
L k_{x}^{2}+M\left(k_{y}^{2}+k_{z}^{2}\right) & N k_{x} k_{y} & N k_{z} k_{x} \\
N k_{x} k_{y} & L k_{y}^{2}+M\left(k_{z}^{2}+k_{x}^{2}\right) & N k_{y} k_{z} \\
N k_{z} k_{x} & N k_{y} k_{z} & L k_{z}^{2}+M\left(k_{x}^{2}+k_{y}^{2}\right)
\end{array}\right] \begin{aligned}
& x \\
& y \\
& z
\end{aligned}
$$

where $m$ is the free electron mass. The dimensionless terms $L, M$, and $N$ are related to the Luttinger $\gamma$ parameters:

$$
\begin{aligned}
& L=\gamma_{1}+4 \gamma_{2}, \\
& M=\gamma_{1}-2 \gamma_{2}, \\
& N=6 \gamma_{3} .
\end{aligned}
$$

The spin-orbit interaction, which is included by adding $H_{s o}$ to $H_{k p}$, is given by the Hamiltonian ${ }^{17}$

$$
H_{s o}=\frac{\Delta_{0}}{3}\left[\begin{array}{cccccc|c}
0 & -i & 0 & 0 & 0 & 1 \\
i & 0 & 0 & 0 & 0 & -i \\
0 & 0 & 0 & -1 & i & 0 & x \uparrow \\
0 & 0 & -1 & 0 & i & 0 & y \uparrow \\
0 & 0 & -i & -i & 0 & 0 & z \uparrow \\
1 & i & 0 & 0 & 0 & 0
\end{array}\right],
$$

where $\Delta_{0}$ is the zone center $(k=0)$ spin-orbit splitting.

Although a numerical eigensolution to the full Hamiltonian [Eq. (5)] was used in this work, an approximation helps in understanding the bearing of the Luttinger parameters on the anisotropy. This approximation is

$$
\begin{aligned}
E= & -\frac{\hbar^{2}}{2 m_{0}}\left[\gamma_{1} k^{2}\right. \\
& \left. \pm \sqrt{4 \gamma_{2}^{2} k^{4}+12\left(\gamma_{3}^{2}-\gamma_{2}^{2}\right)\left(k_{x}^{2} k_{y}^{2}+k_{y}^{2} k_{z}^{2}+k_{z}^{2} k_{x}^{2}\right)}\right],
\end{aligned}
$$

which is valid only if $E \ll \Delta_{0}$. From this, it can be seen that varying $\gamma_{2}$ alone controls the separation between the heavy and light hole bands and that varying $\left(\gamma_{3}^{2}-\gamma_{2}^{2}\right)$ controls the anisotropy.

The eigenvectors of the Hamiltonian in Eq. (5) are obtained numerically, and are used in the calculation of the scattering matrix elements. Thus the scattering rates also reflect the anisotropy of the valence band. For the present case, in pure silicon, the scattering mechanisms used are acoustic and nonpolar optical phonon scattering.

In this work, the noise parameters of concern are the noise temperature, the differential mobility, and the diffusivity, both longitudinal and transverse. The noise temperature is derived from the calculated values of the diffusivity and the differential mobility, as given in Eq. (4). For the longitudinal noise temperature, the appropriate quantities are the longitudinal differential mobility and the longitudinal diffusivity. For the transverse noise temperature, the appropriate quantities are the transverse differential mobility and the transverse diffusivity. Both quantities must refer to the same direction transverse to the electric field since the energy surfaces are warped.

The longitudinal differential mobility is calculated as the slope of the velocity-field characteristics obtained from the Monte Carlo simulation. The transverse differential mobility is calculated, as described in Ref. 9, by adding a small transverse electric field component to the high field and taking the ratio of the resulting transverse drift velocity to the transverse electric field.

The diffusivity tensor is calculated from the formula ${ }^{18}$

$$
D_{i j}=\frac{1}{2} \frac{d}{d t}\left\langle\Delta x_{i} \Delta x_{j}\right\rangle
$$

where $\Delta x_{i}=x_{i}-\overline{x_{i}}$ is the position fluctuation in direction $i$, $i=1,2,3$ (i.e., $x, y, z$ ). The angular brackets denote averaging over the carrier population.

As is the case for all other high-field transport parameters, the noise characteristics have 48 -fold symmetry with respect to the crystallographic orientation of the electric field in the unstrained semiconductor. If the parameters are specified for fields whose directions lie in the sector bounded by the [001], [111], and [101] directions, then they are completely specified. With respect to direction in the plane perpendicular to the electric field, the symmetry of the transverse noise parameters depends upon the orientation of the electric field. ${ }^{9}$ If the field is oriented in the [100] or [111] direction, the transverse parameters are isotropic in their plane. If the field is oriented in the [101] direction, then the parameters are twofold symmetric, with principal axes in the [101] and [010] directions. 


\section{RESULTS}

\section{A. Procedures}

The approach taken in this work is to calculate noise characteristics for silicon and then to compare these results against those obtained for hypothetical materials whose band structure is similar to that of silicon (as reflected in the density of states), but with varying degrees of anisotropy. Specifically, anisotropy is varied by varying the Luttinger parameters in two different ways. First, the value of the Luttinger parameter $\gamma_{1}$ is held fixed at its value for silicon, while $\gamma_{2}$ is independently set to selected values and $\gamma_{3}$ is varied to achieve the same heavy hole density of states, as in silicon, in the range $0-100 \mathrm{meV}$. Second, the value of $\gamma_{2}$ is held fixed at its value for silicon, while $\gamma_{3}$ is independently set to selected values and $\gamma_{1}$ is varied to achieve the same density of states, as in silicon, over the same range as before. In the first case, three sets of Luttinger parameters are used, corresponding to hypothetical materials referred to as materials $\mathrm{A}, \mathrm{B}$, and $\mathrm{C}$. In the second case, three different sets of Luttinger parameters are used, correspondingly referred to as materials $\mathrm{D}, \mathrm{E}$, and $\mathrm{F}$. The variation of the noise characteristics between materials $\mathrm{A}-\mathrm{B}-\mathrm{Si}-\mathrm{C}$ and between materials $\mathrm{D}-\mathrm{E}-\mathrm{Si}-\mathrm{F}$ shows the extent to which anisotropy affects these room temperature transport coefficients.

For each material, three types of Monte Carlo calculation are performed: longitudinal differential mobility, transverse differential mobility, and diffusivity tensor. Each of these quantities is obtained for electric fields of $50 \mathrm{kV} / \mathrm{cm}$ and oriented in each of the three directions [100], [101], and [111]. In the case of transverse differential mobility, the transverse directions used are [010] (for a field in the [100] direction), [211] (for a field in the [111] direction), and [101] and [010] (for a field in the [101] direction). In the first two cases, as stated earlier, any direction in the plane perpendicular to the electric field is symmetrically equivalent to any other direction, and the chosen transverse direction is arbitrary. In the last case, a field oriented in the [101] direction, two orthogonal directions are not equivalent to one another. This requires separate calculation of the transverse characteristics in each of these two directions.

To calculate the longitudinal differential mobility, the velocity-field relation is calculated for a selected set of electric fields over the range of $0-75 \mathrm{kV} / \mathrm{cm}$. Next, the phenomenological function ${ }^{19} v=v_{m} E /\left(E_{c}+E\right)$ is fit to the calculated data. Then the slope taken at $50 \mathrm{kV} / \mathrm{cm}$ to give the longitudinal differential mobility is

$$
\mu_{l}=\frac{v_{m}}{E_{c}}\left(\frac{1}{\left(1+E / E_{c}\right)^{2}}\right) .
$$

To calculate the transverse differential mobility, a relatively small field vector of $2 \mathrm{kV} / \mathrm{cm}$ that is perpendicular to the large $50 \mathrm{kV} / \mathrm{cm}$ electric field vector is added to the $50 \mathrm{kV} / \mathrm{cm}$ vector. The drift velocity is calculated and the component of the velocity that is aligned with the small $2 \mathrm{kV} / \mathrm{cm}$ field component is determined. The ratio of the perpendicular velocity and field components gives the transverse differential mobility in this specific transverse direction:

$$
\mu_{t}=v_{t} / E_{t} .
$$

Earlier work has demonstrated that the transverse velocitytransverse field relationship is linear for the field magnitudes used here, in silicon, ${ }^{9}$ so that, although the calculation is of a chordal mobility, it is equal to the differential mobility in these circumstances.

To calculate the diffusivity tensor elements, the Monte Carlo performs an ensemble simulation to obtain the position estimators $\left\langle x_{i} x_{j}\right\rangle, i, j=1,2,3$, and $\left\langle x_{i}\right\rangle, i=1,2,3$. The brackets denote averaging over all particles of the simulation, which typically involves 50000 particles. After completion of the simulation, the diffusion tensor elements are calculated as

$$
\begin{aligned}
D_{i j}=\frac{1}{2} \frac{d}{d t}\left(\left\langle x_{i} x_{j}\right\rangle-\left\langle x_{i}\right\rangle\left\langle x_{j}\right\rangle\right) & =\frac{1}{2} \frac{d}{d t}\left\langle\left(x_{i}-\bar{x}_{i}\right)\left(x_{j}-\bar{x}_{j}\right)\right\rangle \\
& =\frac{1}{2} \frac{d}{d t}\left\langle\delta x_{i} \delta x_{j}\right\rangle .
\end{aligned}
$$

This derivative must be taken where $\left\langle\delta x_{i} \delta x_{j}\right\rangle$ is linear to obtain a steady-state value of the diffusivity. To accomplish this, a linear, least-squares fit is made to $\left\langle\delta x_{i} \delta x_{j}\right\rangle$ vs $t$. Half of the slope of this line equals the diffusivity. Finally, in order to obtain the diffusivity in the direction of unit vector $\hat{n}=n_{1} \hat{x}+n_{2} \hat{y}+n_{3} \hat{z}$, the dyadic sum is taken:

$$
D_{n}=\sum_{i, j=1}^{3} n_{i} D_{i j} n_{j}
$$

Since the mobilities are derived from the Monte Carlo calculated velocities, uncertainty estimates for the mobilities are based on the uncertainties of the velocities. To estimate the uncertainty in a given component of the drift velocity vector, the value of the velocity component is output periodically during the entire Monte Carlo simulation. Then, after completion of the simulation, this data may be graphed as a velocity versus time plot, which will show an initial, transient behavior, followed by a diminishing fluctuation that approaches the final value from the simulation. An estimate of the uncertainty in the value of the velocity component is obtained from the standard deviation of this velocity versus time data, omitting the initial transient portion. This procedure applies to both the transverse and longitudinal velocities.

Since the transverse differential mobility, $\mu_{t}$, is simply the ratio of the transverse velocity to the transverse electric field, the estimated uncertainty in $\mu_{t}$ is $\delta \mu_{t}=\delta\left(v_{t} / E_{t}\right)$ $=\delta v_{t} / E_{t}$, where $\delta v_{t}$ is the uncertainty in the transverse velocity component.

The process of taking a derivative of the velocity with respect to electric field to obtain the longitudinal differential mobility involves several points on the velocity-field characteristic. In order to obtain an estimated uncertainty of the longitudinal mobility, the velocity uncertainties are obtained at each electric field value for which the carrier velocity has been calculated by Monte Carlo. Then the following procedure is carried out.

(1) At each of the points, a velocity is randomly picked within its estimated range of uncertainty. 
TABLE I. Luttinger valence band structure parameters for silicon and for the six hypothetical materials A-F studied in this work.

\begin{tabular}{cccc}
\hline \hline Material & $\gamma_{1}$ & $\gamma_{2}$ & $\gamma_{3}$ \\
\hline $\mathrm{Si}$ & 4.22 & 0.39 & 1.44 \\
$\mathrm{~A}$ & 4.22 & 1.67 & 1.67 \\
$\mathrm{~B}$ & 4.22 & 1.00 & 1.61 \\
$\mathrm{C}$ & 4.22 & 0.00 & 1.32 \\
$\mathrm{D}$ & 1.66 & 0.39 & 0.39 \\
$\mathrm{E}$ & 3.03 & 0.39 & 1.00 \\
$\mathrm{~F}$ & 5.80 & 0.39 & 2.00 \\
\hline \hline
\end{tabular}

(2) The function $v=v_{m} E /\left(E_{c}+E\right)$ is fit to the resulting set of points.

(3) The slope of the fitting function is calculated at $50 \mathrm{kV} / \mathrm{cm}$.

This procedure is repeated for a large number of iterations (on the order of 10000 ), for each of which, the velocities are randomly varied within the range of uncertainty at each data point. After a large number of repetitions, a range in the resulting slope is established, and this serves as an estimate of the uncertainty of the longitudinal differential mobility.

The uncertainty in the diffusivity is estimated by calculating the range of slopes of $\left\langle\delta x_{i} \delta x_{j}\right\rangle$ vs $t$ resulting from fluctuations in the $\left\langle\delta x_{i} \delta x_{j}\right\rangle$ vs $t$ data. These fluctuations are assumed to fall in the same range in the linear portion of the $\left\langle\delta x_{i} \delta x_{j}\right\rangle$ vs $t$ characteristic. An estimate of a typical fluctuation is obtained as the standard deviation of the $\left\langle\delta x_{i} \delta x_{j}\right\rangle$ data from the linear least-squares fit. Varying the end points of the straight line fit by plus or minus this typical fluctuation and taking the slope of the resulting line gives an estimate of the range of uncertainty in the slope of $\left\langle\delta x_{i} \delta x_{j}\right\rangle$ vs $t$. Half of this estimate equals the uncertainty in the diffusivity, $D_{i j}$.
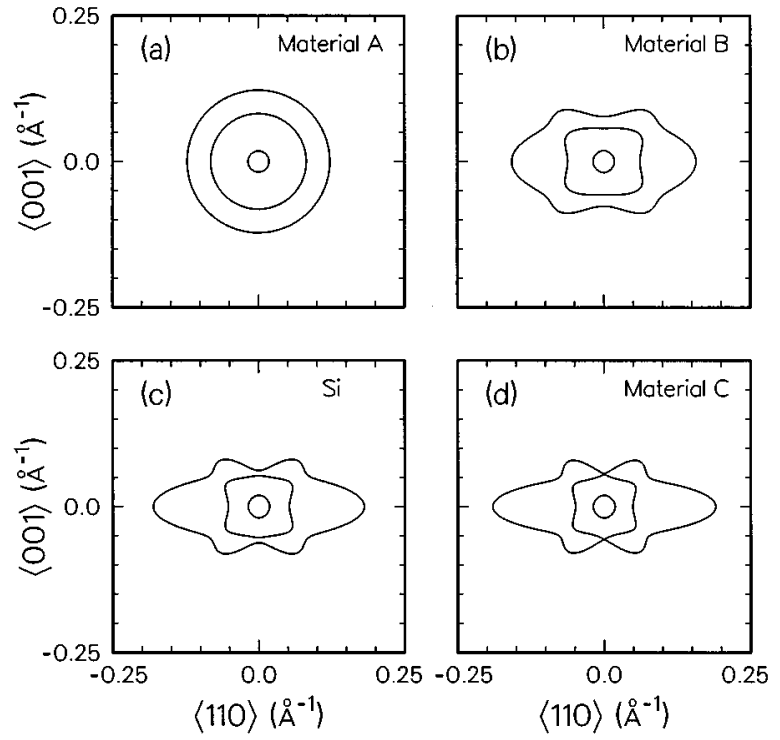

FIG. 1. Constant energy contours for $\mathrm{Si}$ and hypothetical materials $\mathrm{A}, \mathrm{B}$, and $\mathrm{C}$ at $E=50 \mathrm{meV}$ in the $(1 \overline{10})$ plane. These are obtained numerically as eigenvalues of the $\mathbf{k} \cdot \mathbf{p}$ and spin-orbit Hamiltonians [Eq. (5)] using the Luttinger parameters given in Table I.
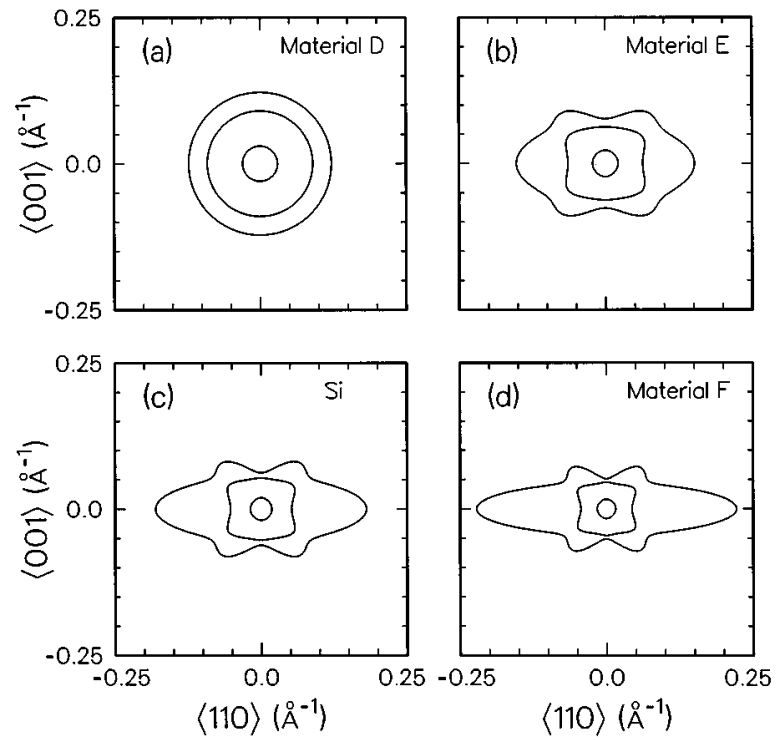

FIG. 2. Constant energy contours for $\mathrm{Si}$ and hypothetical materials D, E, and $\mathrm{F}$ at $E=50 \mathrm{meV}$ in the $(1 \overline{10})$ plane. These are obtained numerically as eigenvalues of the $\mathbf{k} \cdot \mathbf{p}$ and spin-orbit Hamiltonians [Eq. (5)] using the Luttinger parameters given in Table I.

The noise temperature is proportional to the ratio of the diffusivity to the differential mobility, as given in Eq. (4). The relative uncertainty in the noise temperature, $\delta T_{n} / T_{n}$, may be estimated from the relative uncertainties of the diffusivity, $\delta D / D$, and the mobility, $\delta \mu / \mu$, as

$$
\frac{\delta T_{n}}{T_{n}}=\sqrt{\left(\frac{\delta D}{D}\right)^{2}+\left(\frac{\delta \mu}{\mu}\right)^{2}} .
$$

\section{B. Results}

The Luttinger parameters used for silicon and for materials $\mathrm{A}-\mathrm{F}$ are summarized in Table I. Constant energy contours are shown in Figs. 1 and 2. Materials A and D are

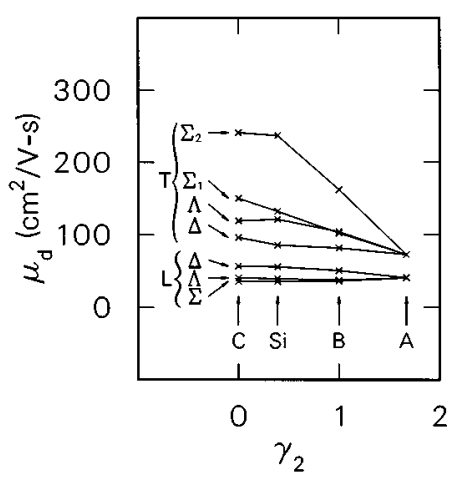

FIG. 3. Longitudinal (L) and transverse (T) high-field differential mobility for $\mathrm{Si}$ and hypothetical materials $\mathrm{A}, \mathrm{B}$, and $\mathrm{C}$ for $T=300 \mathrm{~K}$ and $E=50$ $\mathrm{kV} / \mathrm{cm}$. Data are plotted with respect to the independently varied Luttinger parameter $\gamma_{2}$. Each curve corresponds to a specific electric field orientation: $\Delta-E\|[100], \Lambda-E\|[111], \Sigma-E \|[101]$. For the transverse $\Sigma$ data, additional labeling denotes the specific transverse direction: $\Sigma_{1}-\hat{e}_{t} \|[010]$, $\Sigma_{2}-\hat{e}_{t} \|[\overline{101}]$. Material A is isotropic, B is anisotropic, but less so than silicon, and $\mathrm{C}$ is more anisotropic than silicon. 


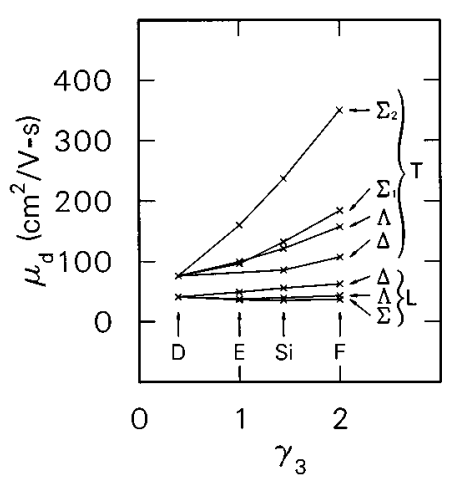

FIG. 4. Longitudinal (L) and transverse (T) high-field differential mobility for $\mathrm{Si}$ and hypothetical materials $\mathrm{D}, \mathrm{E}$, and $\mathrm{F}$ for $T=300 \mathrm{~K}$ and $E=50$ $\mathrm{kV} / \mathrm{cm}$. Data are plotted with respect to the independently varied Luttinger parameter $\gamma_{3}$. Curves are labeled as in Fig. 3. Material D is isotropic, E is anisotropic, but less so than silicon, and $\mathrm{F}$ is more anisotropic than silicon.

isotropic. Materials B and E are anisotropic, but less so than silicon. Materials $\mathrm{C}$ and $\mathrm{F}$ are more anisotropic than silicon.

The differential mobilities for silicon and materials $\mathrm{A}-\mathrm{C}$ are shown in Fig. 3 and for silicon and materials D-F, in Fig. 4. In Figs. 3 and 4, the three curves labeled ' $L$ ' are the longitudinal differential mobility and the four curves labeled " $\mathrm{T}$ ' are the transverse differential mobility. The orientations of the electric field are denoted $\Delta$ ([100]), $\Sigma$ ([101]), and $\Lambda$ ([111]). In the case of the transverse [101] data, the two nonequivalent transverse directions are labeled $\Sigma_{1}([010])$ and $\Sigma_{2}([\overline{101}])$. The diffusivities for silicon and materials $\mathrm{A}-\mathrm{C}$ are shown in Fig. 5, and for silicon and materials D-F in Fig. 6. The labeling nomenclature is the same as that used for the mobility. Noise temperatures are shown in Figs. 7 ( $\mathrm{Si}$, $\mathrm{A}-\mathrm{C})$ and $8(\mathrm{Si}, \mathrm{D}-\mathrm{F})$, with labeling as before.

Error analysis, as described above, yields the following estimates for uncertainties in the differential mobilities, diffusivities, and noise temperatures: $\delta \mu_{l} / \mu_{l} \leqslant 0.2 \%$, $\delta \mu_{t} / \mu_{t} \leqslant 6.1 \%, \quad \delta D_{l, t} / D_{l, t} \leqslant 0.4 \%, \quad \delta T_{n l} / T_{n l} \leqslant 0.4 \%, \quad$ and $\delta T_{n t} / T_{n t} \leqslant 6.1 \%$.

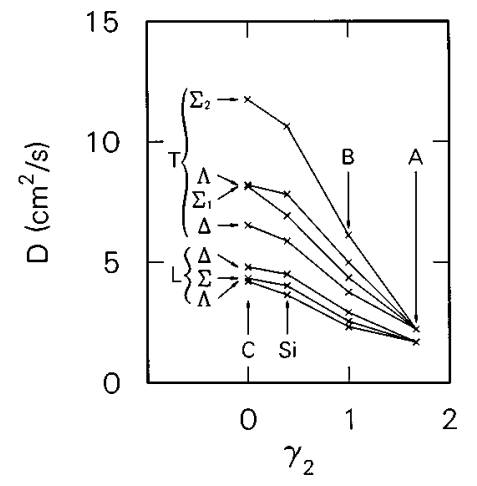

FIG. 5. Longitudinal (L) and transverse (T) high-field diffusivity for Si and hypothetical materials A, B, and C for $T=300 \mathrm{~K}$ and $E=50 \mathrm{kV} / \mathrm{cm}$. Data are plotted with respect to the independently varied Luttinger parameter $\gamma_{2}$. Labeling is the same as in Fig. 3.

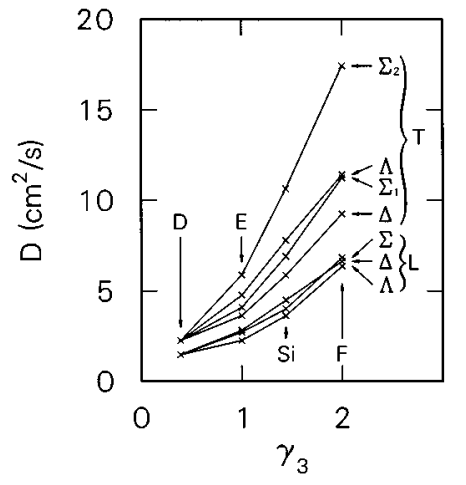

FIG. 6. Longitudinal (L) and transverse (T) high-field diffusivity for Si and hypothetical materials D, E, and F for $T=300 \mathrm{~K}$ and $E=50 \mathrm{kV} / \mathrm{cm}$. Data are plotted with respect to the independently varied Luttinger parameter $\gamma_{3}$. Labeling is the same as in Fig. 4.

\section{Analysis}

The anisotropy of the longitudinal differential mobility in silicon is straightforwardly derived from the familiar variations of effective mass between the three directions shown. An analysis of the anisotropy of the transverse differential mobility in silicon was given in Ref. 9. To summarize those findings, the variation of the transverse mobility directly correlates to the variation of the conductivity effective mass in the given transverse direction when a high electric field is being applied. The effect of the high field is to shift the center of the hole distribution away from the Brillouin zone center, causing the particles to experience a different effective mass, on average, than they would were they centered about $k=0$. As shown in Figs. 3 and 4, the distinctions between the mobilities associated with the different field orientations arise from the valence band anisotropy and therefore increase monotonically with the increase in anisotropy.

The increase of the diffusivities with respect to valence band anisotropy, shown in Figs. 5 and 6, is caused by sampling of a wider range of conductivity effective masses by the holes. To understand this, let us consider the longitudinal diffusivity labeled $\Delta$ in Fig. 5. The electric field is oriented in

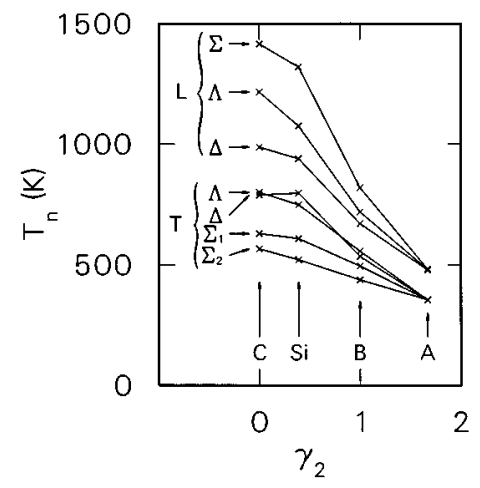

FIG. 7. Longitudinal (L) and transverse (T) high-field noise temperature for Si and hypothetical materials A, B, and C for $T=300 \mathrm{~K}$ and $E=50 \mathrm{kV} / \mathrm{cm}$. Data are plotted with respect to the independently varied Luttinger parameter $\gamma_{2}$. Labeling is the same as in Fig. 3. 


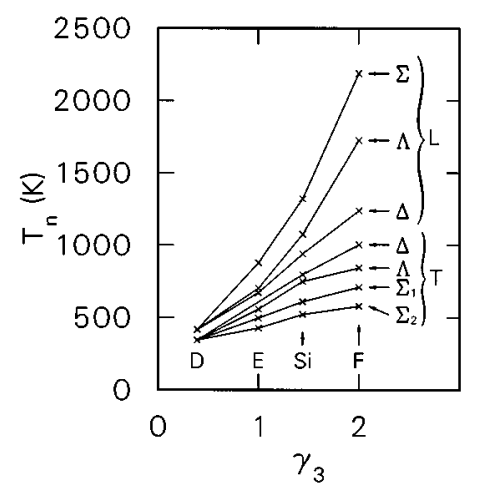

FIG. 8. Longitudinal (L) and transverse (T) high-field noise temperature for $\mathrm{Si}$ and hypothetical materials $\mathrm{D}, \mathrm{E}$, and $\mathrm{F}$ for $T=300 \mathrm{~K}$ and $E=50 \mathrm{kV} / \mathrm{cm}$. Data are plotted with respect to the independently varied Luttinger parameter $\gamma_{3}$. Labeling is the same as in Fig. 4.

the [100] direction in this case. For a single energy, the lateral spread of the reciprocal-space hole distribution causes the holes to experience not just a single effective mass, as in the case of an isotropic band structure but, rather, a range of conductivity effective masses due to the warping of the valence band constant energy surfaces. This geometrical mass variation increases the fluctuation of velocities, and thereby the diffusivity, in the same way that intervalley scattering does in multivalley conduction band transport.

The dependence of the noise temperature on band structure anisotropy can be understood in terms of the behavior of its constituents of mobility and diffusivity. First, as discussed above, the diffusivities, both longitudinal and transverse, increase with anisotropy. But, while the transverse differential mobility also increases pronouncedly with band anisotropy, the longitudinal differential mobility exhibits only a slight increase $(\Delta)$ or even a decrease $(\Sigma$ and $\Lambda)$ with respect to anisotropy. This is a consequence of the onset of velocity saturation. The result is that, as anisotropy is increased, the transverse small signal impedance decreases due to the lowering of transverse effective masses. This is sufficient, in a siliconlike material, to significantly offset the diffusivity increase, resulting in only a modest increase of the transverse noise temperature or, equivalently, the transverse available noise power. The result, however, for the longitudinal noise power is that, due primarily to the increase in the longitudinal small signal impedance, and supported by the general diffusivity increase with anisotropy, the longitudinal noise temperature and the longitudinal available noise power increase markedly with band structure anisotropy.

\section{DISCUSSION}

The following conclusions can be drawn from this work. First of all, high-field noise temperatures, both longitudinal and transverse, increase with valence band anisotropy. The longitudinal increase is substantial, however, compared to the transverse noise temperature increase. The difference between the two depends on the specific orientation of the electric field. Second, isotropic materials have only negligible excess transverse noise temperature and a minimum excess longitudinal noise temperature.
On a speculative note, high-field thermal noise measurements may provide an alternative to magnetotransport measurements for determining valence band structure in large band gap semiconductors such as diamond and cubic GaN. Supporting this is the pronounced dependence on band structure features of both the transverse differential mobility and the longitudinal noise temperature. Both of these quantities are measurable at room temperature using well established microwave techniques.

From the data shown in Figs. 3 and 4, the greatest transverse mobility (or conductivity) sensitivity to the shape of the band structure occurs for fields in the [101] orientation with the transverse measurement taken in the [101] $\left(\Sigma_{2}\right)$ direction. Measurement uncertainties, therefore, would have the least effect on the determination of the Luttinger parameters for measurements taken in this orientation. Similarly, from the data shown in Figs. 7 and 8, the determination of Luttinger parameters from measurements of longitudinal noise temperature is least susceptible to measurement uncertainties for measurements taken in the [101] $\left(\Sigma_{2}\right)$ direction, due to the strongest variation of $T_{n l}$ with respect to $\gamma_{2}$ and $\gamma_{3}$, for fields along the [101] axis.

Regarding the choice of semiconductor materials and crystallographic orientations of biasing fields in devices, the data in Figs. 7 and 8 indicate that, from a noise suppression standpoint, the best orientation of the electric field is the [100] direction, this having the lowest longitudinal available noise power. Fortunately, this is the same orientation that gives the maximum carrier mobility so that high speed and low noise are achieved simultaneously. The data in these figures also show that, where a choice can be made between different semiconductor materials, a lower hole current thermal noise will be achieved by using the material with the least valence band anisotropy.

\section{ACKNOWLEDGMENTS}

This work was supported by the U.S. Air Force (Grant No. F49620-95-1-0013) and by the U.S. Army URI program (Grant No. DAAL03-92-G-0109).

${ }^{1}$ J. M. Luttinger, Phys. Rev. 102, 1030 (1956).

${ }^{2}$ B. Lax and J. G. Mavroides, Solid State Physics, edited by F. Seitz and D. Turnbull (Academic, New York, 1960), Vol. 11, p. 261.

${ }^{3}$ J. C. Hensel and G. Feher, Phys. Rev. 129, 1041 (1963).

${ }^{4}$ S. Weinreb and A. R. Kerr, IEEE J. Solid-State Circuits 8, 58 (1973).

${ }^{5}$ J. P. Nougier, NATO ASI Ser. B 52, 415 (1980).

${ }^{6}$ Y. K. Pozhela, Hot-Electron Transport in Semiconductors, edited by L. Reggiani (Springer, Berlin, 1984), Chap. 4.

${ }^{7}$ V. Bareikis, R. Katilius, A. Matulionis, R. Saltis, S. Gantsevich, V. Gurevich, S. Kogan, and A. Shulman, Lith. Phys. J. 31, 3 (1991).

${ }^{8}$ L. Reggiani, T. Kuhn, and L. Varani, Appl. Phys. A 54, 411 (1992).

${ }^{9}$ J. M. Hinckley and J. Singh, Appl. Phys. Lett. 67, 2966 (1995).

${ }^{10}$ J. M. Hinckley and J. Singh, Appl. Phys. Lett. 66, 2727 (1995).

${ }^{11}$ A. Van der Ziel, Noise in Solid State Devices and Circuits (Wiley, New York, 1986).

${ }^{12}$ J. M. Hinckley and J. Singh, Phys. Rev. B 42, 3546 (1990).

${ }^{13}$ J. M. Hinckley and J. Singh, J. Appl. Phys. 76, 4192 (1994).

${ }^{14}$ J. M. Hinckley, Ph.D. thesis, University of Michigan, Ann Arbor, 1990.

${ }^{15}$ J. M. Hinckley and J. Singh, Phys. Rev. B 41, 2912 (1990).

${ }^{16}$ G. Dresselhaus, A. F. Kip, and C. Kittel, Phys. Rev. 98, 368 (1955).

${ }^{17}$ M. Tiersten, IBM J. Res. Dev. 5, 122 (1961).

${ }^{18}$ C. Jacoboni and L. Reggiani, Rev. Mod. Phys. 55, 645 (1983).

${ }^{19}$ D. M. Caughey and R. E. Thomas, Proc. IEEE 14, 2192 (1967). 